\title{
Climatology of rapid geomagnetic variations at high latitudes over two solar cycles
}

\author{
A. Viljanen ${ }^{1}$ and E. Tanskanen ${ }^{1,2}$ \\ ${ }^{1}$ Finnish Meteorological Institute, P.O. Box 503, 00101 Helsinki, Finland \\ ${ }^{2}$ University of Bergen, Dept. of Physics and Technology, Allégatan 55, Bergen 5007, Norway
}

Received: 30 November 2010 - Revised: 17 March 2011 - Accepted: 3 October 2011 - Published: 11 October 2011

\begin{abstract}
We investigate the characteristics of rapid geomagnetic variations at high latitudes based on the occurrence of large time derivatives of the horizontal magnetic field $\left(d \boldsymbol{H} / d t\right.$ exceeding $\left.1 \mathrm{nT} \mathrm{s}^{-1}\right)$. Analysis of IMAGE magnetometer data from North Europe in 1983-2010, covering more than two solar cycles, confirms and specifies several previous findings. We show that $d \boldsymbol{H} / d t$ activity is high around the midnight and early morning hours, and nearly vanishes at noon and early afternoon. This happens during all seasons, although the midnight maximum is nearly invisible during summer. As indicated by modelled ionospheric equivalent currents, large $d \boldsymbol{H} / d t$ values occur predominantly during westward ionospheric electrojets. Before and around midnight, $d \boldsymbol{H} / d t$ tends to be north-south oriented, whereas in the morning hours, its direction is more west-east directed. $d \boldsymbol{H} / d t$ tends to be more strictly north-south oriented during winter than other seasons. The seasonal occurrence of large $d \boldsymbol{H} / d t$ values is similar to the variation of the maximum amplitude of westward equivalent currents. The yearly fraction of east-west directed large $d \boldsymbol{H} / d t$ vectors at the Kilpisjärvi station (MLAT 65.88) varies from 31 to $47 \%$ without any clear correlation with the general geomagnetic activity nor with the yearly averages of solar wind parameters.
\end{abstract}

Keywords. Geomagnetism and paleomagnetism (Rapid time variations)

\section{Introduction}

Rapid geomagnetic variations have practical significance, since they drive geomagnetically induced currents (GIC) in technological conductor systems (e.g. Watermann, 2007). In most reported cases, the time derivative of the horizontal

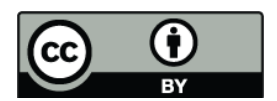

Correspondence to: A. Viljanen (ari.viljanen@fmi.fi) magnetic field $(d \boldsymbol{H} / d t)$ has a high correlation with GIC. This observation follows from the fact that the primary cause of GIC, the geoelectric field, is related to the geomagnetic field via the surface impedance, which acts as a low-pass filter for $d \boldsymbol{H} / d t$. Although modelling of GIC for past events is fairly straightforward (e.g. Pirjola, 2002; Viljanen et al., 2004), forecasting GIC based on physical models from the solar wind down to the ground is still highly challenging (e.g. Pulkkinen et al., 2010). Consequently, analysis of past events will probably provide guidelines for improving forecast methods.

Large GIC events occur most frequently at high latitudes in the vicinity of auroral electrojets and other dynamic ionospheric current systems. Viljanen et al. (2001) showed by using the IMAGE magnetometer data from North Europe in 1982-2001 that large $d \boldsymbol{H} / d t$ (exceeding $1 \mathrm{nT} \mathrm{s}^{-1}$ ) primarily occur during night-time events governed by westward ionospheric currents. However, the directional distributions of $d \boldsymbol{H} / d t$ are much more scattered than those of the simultaneous baseline subtracted horizontal variation field vector $\boldsymbol{H}$. Another study restricted to 833 selected substorms in 1997 and 1999, before a solar maximum, showed a similar behaviour (Viljanen et al., 2006). Turnbull et al. (2009) performed a corresponding investigation for 553 substorms at mid latitudes in 2000-2003 just after a solar maximum. They also found that large $d \boldsymbol{H} / d t$ are generally connected to westward ionospheric currents, but are also affected by smallerscale ionospheric structures.

Using IMAGE magnetometer data of 2002-2003, Pulkkinen et al. (2006) studied spatiotemporal scaling properties of horizontal magnetic field fluctuations in terms of structure function analysis. One result relevant to the present investigation was that the spatial symmetry of field fluctuations increases during substorms, indicating the presence of spatially less ordered ionospheric currents. In distribution patterns of $d \boldsymbol{H} / d t$, this appears as an increasing scatter of directions. 
Table 1. IMAGE magnetometer stations used in this study, and their geographic and corrected geomagnetic coordinates (CGM, year 2001).

\begin{tabular}{lcccc}
\hline Station & Lat & Long & MLAT & MLON \\
\hline SOR (Sørøya) & 70.54 & 22.22 & 67.34 & 106.17 \\
KEV (Kevo) & 69.76 & 27.01 & 66.32 & 109.24 \\
KIL (Kilpisjärvi) & 69.02 & 20.79 & 65.88 & 103.79 \\
MUO (Muonio) & 68.02 & 23.53 & 64.72 & 105.22 \\
PEL (Pello) & 66.90 & 24.08 & 63.55 & 104.92 \\
\hline
\end{tabular}

In this paper, we extend the timeseries used by Viljanen et al. (2001) to ground magnetic field data of 1983-2010 covering more than two solar cycles. Since large $d \boldsymbol{H} / d t$ events typically occur during substorms, we concentrate on analysing data from the high latitude Kilpisjärvi (KIL) station (MLAT 65.88), supplemented by four other nearby sites with a good data coverage. We also determine ionospheric equivalent currents across a long meridional chain to compare quantitatively main similarities and differences between the characteristics of electrojets and high $d \boldsymbol{H} / d t$ activity.

\section{Results}

We start by updating some parts of the analysis of Viljanen et al. (2001) with a more extensive IMAGE magnetometer dataset of 1983-2010 covering completely the solar cycles 22 (1986-1996) and 23 (1997-2008/2009). We will concentrate on results from a selected set of five stations located in the auroral region (Table 1).

We will use the geographic components of the ground magnetic field $\boldsymbol{B}=(X, Y, Z)$, where $X$ points to the north, $Y$ to the east and $Z$ vertically downwards. The vector $\boldsymbol{H}=$ $(X, Y)$ denotes the horizontal part of $\boldsymbol{B}$. For the interplanetary magnetic field (IMF), we use the notation IMF $\boldsymbol{B}=$ $\left(B_{\mathrm{X}}, B_{\mathrm{y}}, B_{\mathrm{Z}}\right)$. We use the magnetic local time (MLT).

The basic dataset consists of all timesteps in 1983-2010 fullfilling the condition that the magnitude of $d \boldsymbol{H} / d t$ exceeds $60 \mathrm{nT} \mathrm{min}^{-1}\left(1 \mathrm{nT} \mathrm{s}^{-1}\right)$. We use 20 -s values similarly to Viljanen et al. (2001). This is the best resolution available for the whole period under study. The time derivatives are calculated as simple differences between successive values divided by $20 \mathrm{~s}$. The quiet time baseline is subtracted from the horizontal field $\boldsymbol{H}$, so it represents the field due to ionospheric currents, with some contribution by the induced currents in the earth. Concerning GIC effects, the origin of the field at the earth's surface is not significant, but only its total value matters. For more details about the effect of induction on the ground magnetic field, see Viljanen et al. (2001) and Tanskanen et al. (2001).

\subsection{Extended analysis of $d \mathrm{H} / \mathrm{dt}$}

The most pronounced difference between the directional distributions of $\boldsymbol{H}$ and $d \boldsymbol{H} / d t$ takes place at about 02:0007:00 MLT when a large number of $d \boldsymbol{H} / d t$ vectors tend to point to the (geomagnetic) east-west direction, whereas $\boldsymbol{H}$ points strictly to the south (Fig. 1). It is evident that a westward current must flow in the ionosphere to produce such $\boldsymbol{H}$. However, the simultaneous distribution patterns of $d \boldsymbol{H} / d t$ cannot be explained by any simple sheet-type current model, but rapidly changing north-south currents and field-aligned currents must play an important role. A comparison to Fig. 9 in Viljanen et al. (2001) based on years 1994-2001 does not indicate any remarkable change with respect to the larger dataset (Viljanen et al., 2001, showed results from SOR, whose latitudinal separation from KIL is about $1.5 \mathrm{deg}$ to the north).

A rough division of $d \boldsymbol{H} / d t$ directions at KIL is shown in Fig. 2. We use two classes: (magnetic) north-south (NS) and east-west (EW). We measure the direction angle $\alpha$ from the geographic north positive clockwise (east). All $d \boldsymbol{H} / d t$ vectors with $-55^{\circ} \leq \alpha \leq+35^{\circ}$ or $125^{\circ} \leq \alpha \leq 215^{\circ}$ belong to the NS sector, and the rest belong to the EW sector. The selected ranges take into account that the geomagnetic north points about 10 degrees counterclockwise from the geographic one in this region. As is evident from Fig. 1, the horizontal variation field $\boldsymbol{H}$ lies nearly only in the NS sector $(85 \%$ of all values). Its time derivative is more likely in the EW sector during the early morning. Of all large $d \boldsymbol{H} / d t$ vectors, $39 \%$ belong to the EW sector.

The results for KIL are quite representative for the whole IMAGE network as we will show next. We considered a chain of 12 stations from Tartu in the south (MLAT 54.5) to Ny Ålesund in the north (MLAT 75.3) in 1994-2009 (in this case, with 1-min data). Of large $d \boldsymbol{H} / d t, 23-52 \%$ belong to the EW sector, but only $11-23 \%$ of $\boldsymbol{H}$ belong to it. As noted by Viljanen et al. (2001), $d \boldsymbol{H} / d t$ is more sensitive to local ground conductivity anomalies than $\boldsymbol{H}$. This is especially visible at station Masi (MLAT 66.2), where $52 \%$ of $d \boldsymbol{H} / d t$ belong to the EW sector. The smallest occurrence of $d \boldsymbol{H} / d t$ in the EW sector takes place in the subauroral region in the southernmost part of IMAGE.

Directional distributions of $d \boldsymbol{H} / d t$ have prominent seasonal variations (Fig. 3), as already found by Viljanen et al. (2001). Especially in winter, $d \boldsymbol{H} / d t$ is more concentrated to the north-south direction than during other seasons. Autumn and spring are very similar to each other. Summer has the most scattered distribution. The occurrence probability of large $d \boldsymbol{H} / d t$ is $1.46 \%$ (spring), $0.82 \%$ (summer), $1.31 \%$ (autumn), $1.02 \%$ (winter). Tanskanen (2009) reports a corresponding seasonal dependence in the peak amplitude of substorms. On the other hand, the number of substorms has its maximum in winter.

As a new result, we present the diurnal occurrence of large $d \boldsymbol{H} / d t$ during different seasons (Fig. 4). Spring and 


\begin{tabular}{c} 
KIL (1983-2009) \\
$\begin{array}{c}\text { directional distribution of } \mathrm{dH} / \mathrm{dt}(\mathrm{dH} / \mathrm{dt}>1 \mathrm{nT} / \mathrm{s}) \\
\text { sampling interval } 20 \mathrm{~s}, 543748 \text { values }\end{array}$ \\
\hline
\end{tabular}
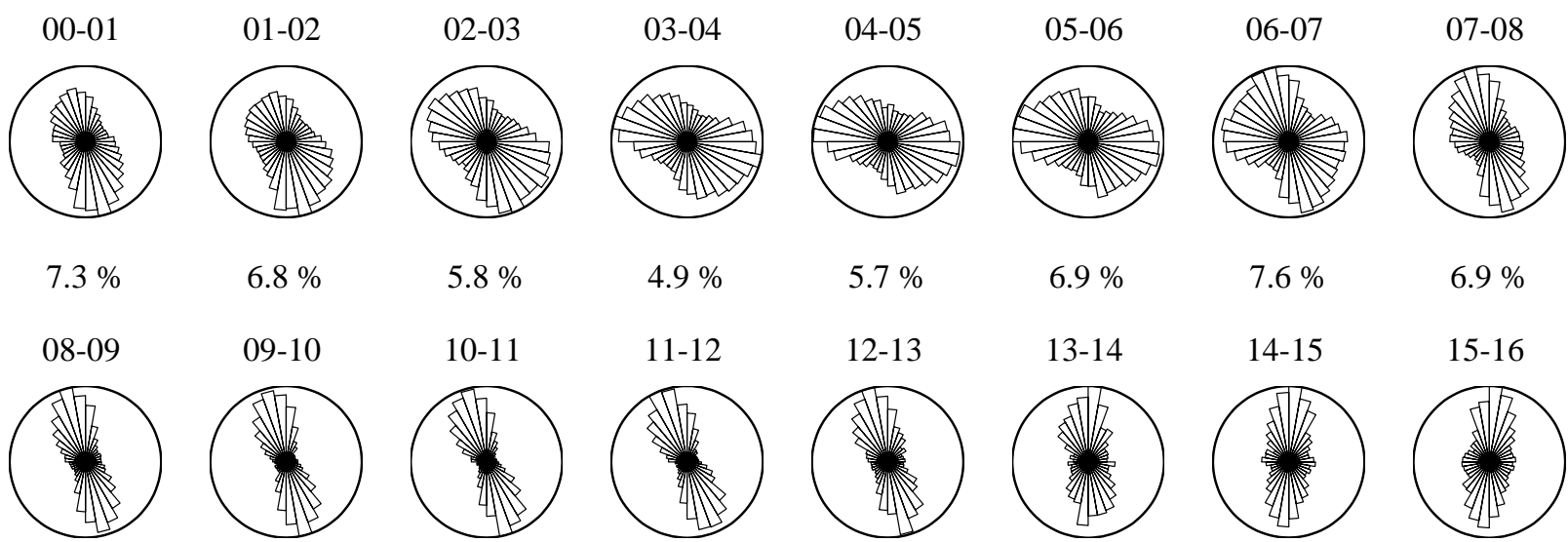

$6.8 \%$

$5.8 \%$

$4.9 \%$

$5.7 \%$

$6.9 \%$

$7.6 \%$

$6.9 \%$

09-10

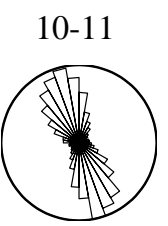

11-12

12-13

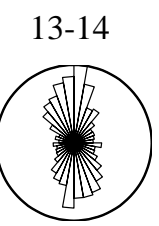

14-15

15-16
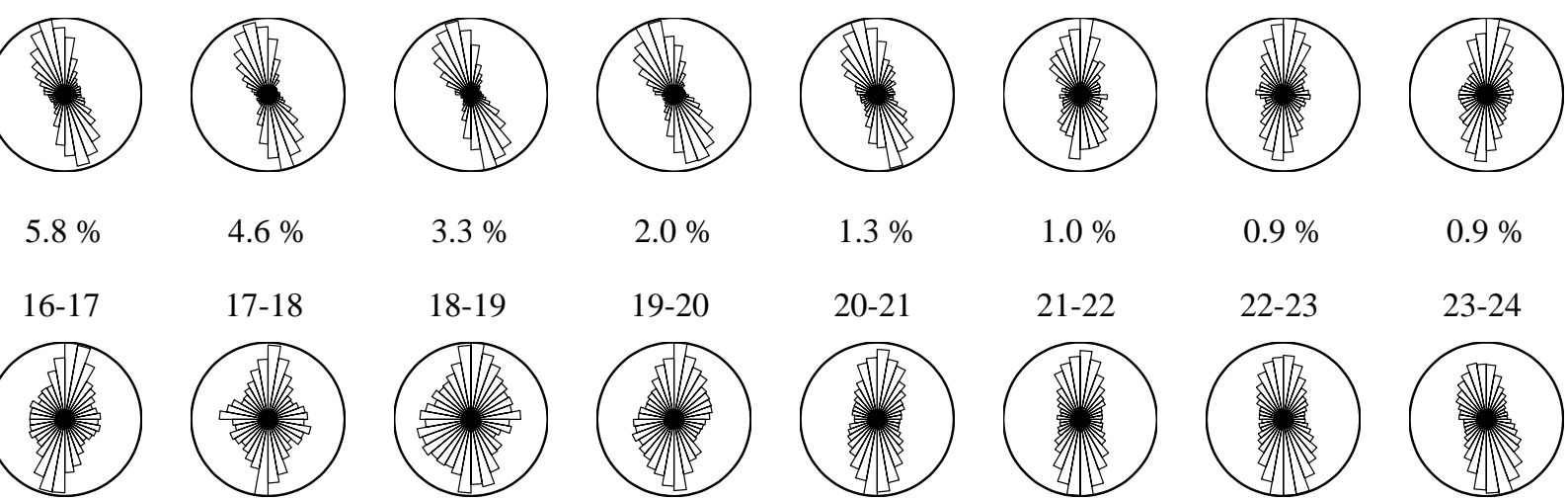

$4.6 \%$

$3.3 \%$

$2.0 \%$

$1.3 \%$

$1.0 \%$

$0.9 \%$

$0.9 \%$

\section{7-18}

18-19

19-20

20-21

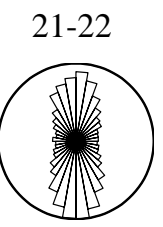

22-23

23-24
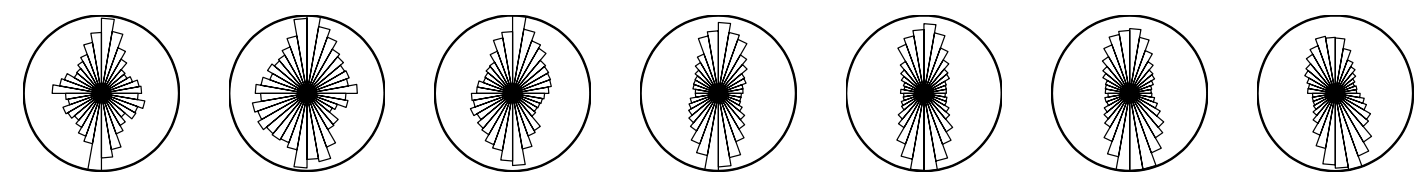

$1.0 \%$

$1.3 \%$

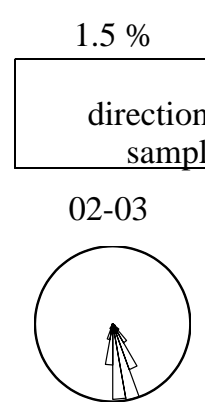

$2.2 \%$

$3.5 \%$

$4.7 \%$

KIL (1983-2009)

$6.4 \%$

$7.7 \%$

rectional distribution of $\mathrm{H}(\mathrm{dH} / \mathrm{dt}>1 \mathrm{nT} / \mathrm{s})$

sampling interval $20 \mathrm{~s}, 543748$ values
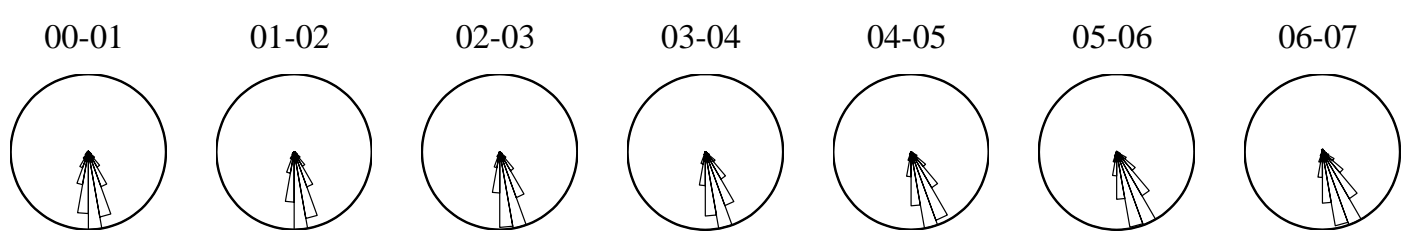

07-08

$7.3 \%$

$6.8 \%$

$5.8 \%$

$4.9 \%$

$5.7 \%$

$6.9 \%$

$7.6 \%$

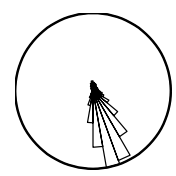

08-09

09-10

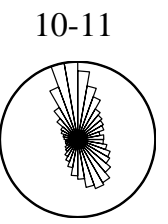

11-12

12-13

13-14

14-15

$6.9 \%$
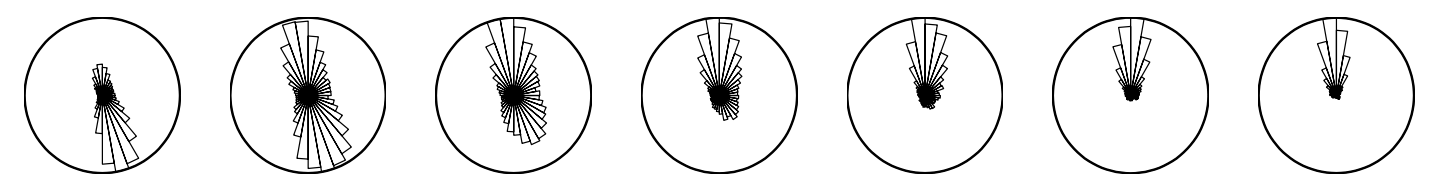

15-16

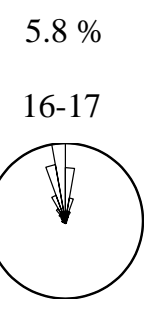

$4.6 \%$

$3.3 \%$

$2.0 \%$

$1.3 \%$

$1.0 \%$

$0.9 \%$

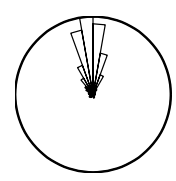

17-18

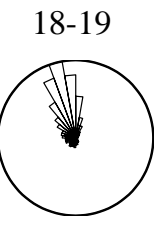

19-20

20-21

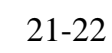

22-23

$0.9 \%$
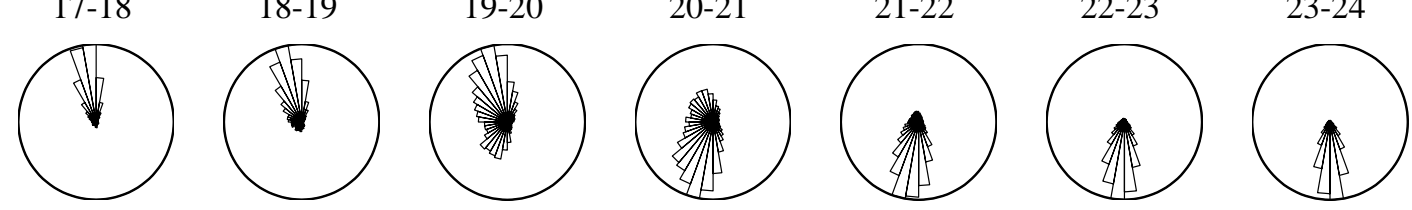

$1.0 \%$

$1.3 \%$

$1.5 \%$

$2.2 \%$

$3.5 \%$

$4.7 \%$

$6.4 \%$

$7.7 \%$

Fig. 1. Hourly directional distributions of $d \boldsymbol{H} / d t$ and $\boldsymbol{H}$ for timesteps with $d \boldsymbol{H} / d t$ exceeding $1 \mathrm{nT} \mathrm{s}{ }^{-1}$ at KIL. The relative number of contributing $d \boldsymbol{H} / d t$ values is given below each diagram. The geographic north is up and east to the right. Magnetic local time is used. 

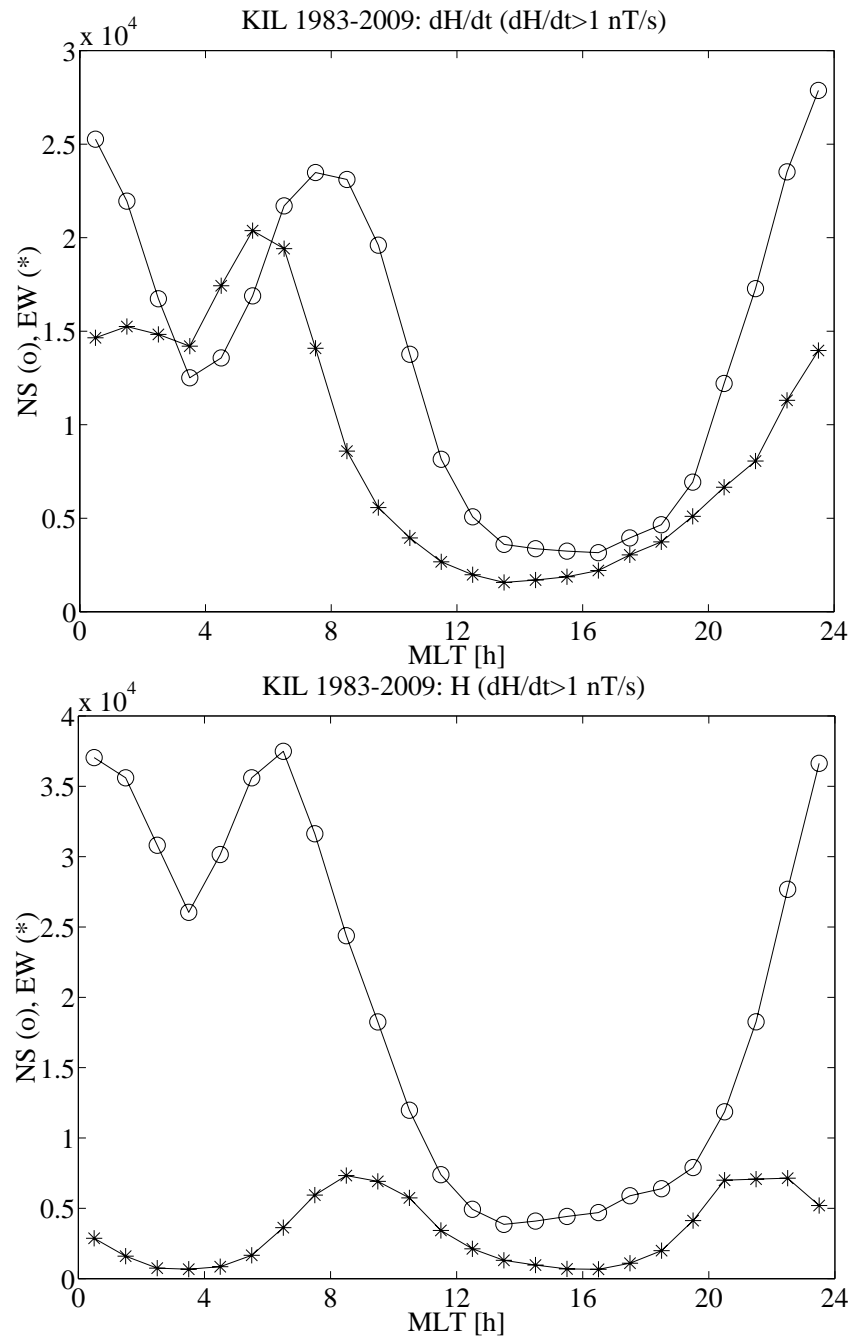

Fig. 2. Upper panel: number of large $d \boldsymbol{H} / d t$ in NS (circles) and EW (asterisks) sectors at KIL (see text for the definitions of the sectors). Lower panel: the same for $\boldsymbol{H}$.

autumn are again very similar to each other: the midnight and morning maxima are equally large. The difference between winter and summer is clear: it is most likely to have large $d \boldsymbol{H} / d t$ around midnight in winter, whereas the morning time is strongly preferred in summer. This is obviously related to the large scatter of directions in summer (Fig. 3), since pulsations are common in the morning (Fig. 1).

The year-to-year variation of the $d \boldsymbol{H} / d t$ distribution and its diurnal occurrence at KIL are illustrated in Fig. 5). In ten years, the midnight maximum is slightly higher (19831985, 1987-1988, 1996, 1998-1999, 2004, 2009). For the rest 18 years, the morning maximum is more pronounced. This behaviour does not seem to depend on the activity level as measured by the yearly number of large $d \boldsymbol{H} / d t$.

As a side note we remark that year 2009 was exceptionally quiet with only $1736 d \boldsymbol{H} / d t$ values exceeding $1 \mathrm{nT} \mathrm{s}^{-1}$ at KIL (Fig. 5). The apparently large scatter in the directional distribution in 2009 may be occasional due to the small number of data points. The low activity in 2008-2009 is clearly related to the general decrease of geomagnetic activity during the somewhat diffuse transition from the solar cycle 23 to cycle 24 (cf. Minamoto and Taguchi, 2009). A clear increase of activity is evident in 2010, although it still remains at a very low level compared to the previous years 1983-2008.

The yearly fraction of EW directed large $d \boldsymbol{H} / d t$ vectors at KIL varies between about 31 and $47 \%$ (Fig. 6). It has some correlation with the solar wind magnetic field. Interestingly, correlation with IMF $B$ is high in 1984-1994, after which it clearly decreases. The same behaviour is found for the alpha particle to proton ratio in the solar wind (plot not shown). Density and dynamic pressure are quite unrelated to the scatter of $d \boldsymbol{H} / d t$.

The solar wind velocity and the $d \boldsymbol{H} / d t$ scatter anticorrelate with each other at a medium level. Years 1994 and 2003 with a very large number of high-speed streams (Table 2) coincide with a small scatter of $d \boldsymbol{H} / d t$. On the other hand, the smallest scatter occurs in 2007 without any high-speed streams and with quite a typical yearly average velocity.

We also remark that the scatter of $d \boldsymbol{H} / d t$ distributions is smallest close to the minimum of a solar cycle, although not necessarily at the exact minimum year. Especially, the minimum in 1994 occurs two years before the solar cycle minimum, and year 1994 was geomagnetically highly active (see Fig. 5). The previous minimum year of the scatter (1985) was much more quiet. This is even more prominent for 2007.

\subsection{Auroral electrojets and $d \mathrm{H} / d t$}

As already noted, the westward electrojet is clearly related to the majority of the large $d \boldsymbol{H} / d t$ values. For a more quantitative analysis, we determined ionospheric equivalent currents for the period of 1994-2010 when the IMAGE magnetometer stations cover the whole auroral region. We applied the method of spherical elementary current systems (Amm and Viljanen, 1999; Pulkkinen et al., 2003) to calculate the (geographic) eastward current density along the meridian $22.1^{\circ} \mathrm{E}$ with a $0.60 \mathrm{deg}$ latitudinal step from 59.0 to $79.4^{\circ} \mathrm{N}$, assuming that the current flow at the altitude of $100 \mathrm{~km}$. We performed the calculation for each day with 1-min data.

As the upper panel of Fig. 7 shows, an eastward electrojet dominates the afternoon hours at about 13:00-21:00 MLT, and a westward current dominates other times having its maximum at about 01:30 MLT. Comparison of this result to the diurnal occurrence of large $d \boldsymbol{H} / d t$ (Fig. 4) shows that there is a general agreement with a high nighttime activity. However, the distinct morning maximum of large $d \boldsymbol{H} / d t$ values around 05:00-09:00 MLT does not have a counterpart with the electrojets. Similarly, there is no high occurrence rate of large $d \boldsymbol{H} / d t$ around the maximum time of the eastward electrojet.

We also determined the daily maximum amplitude of the eastward and westward electrojets, and from these values 

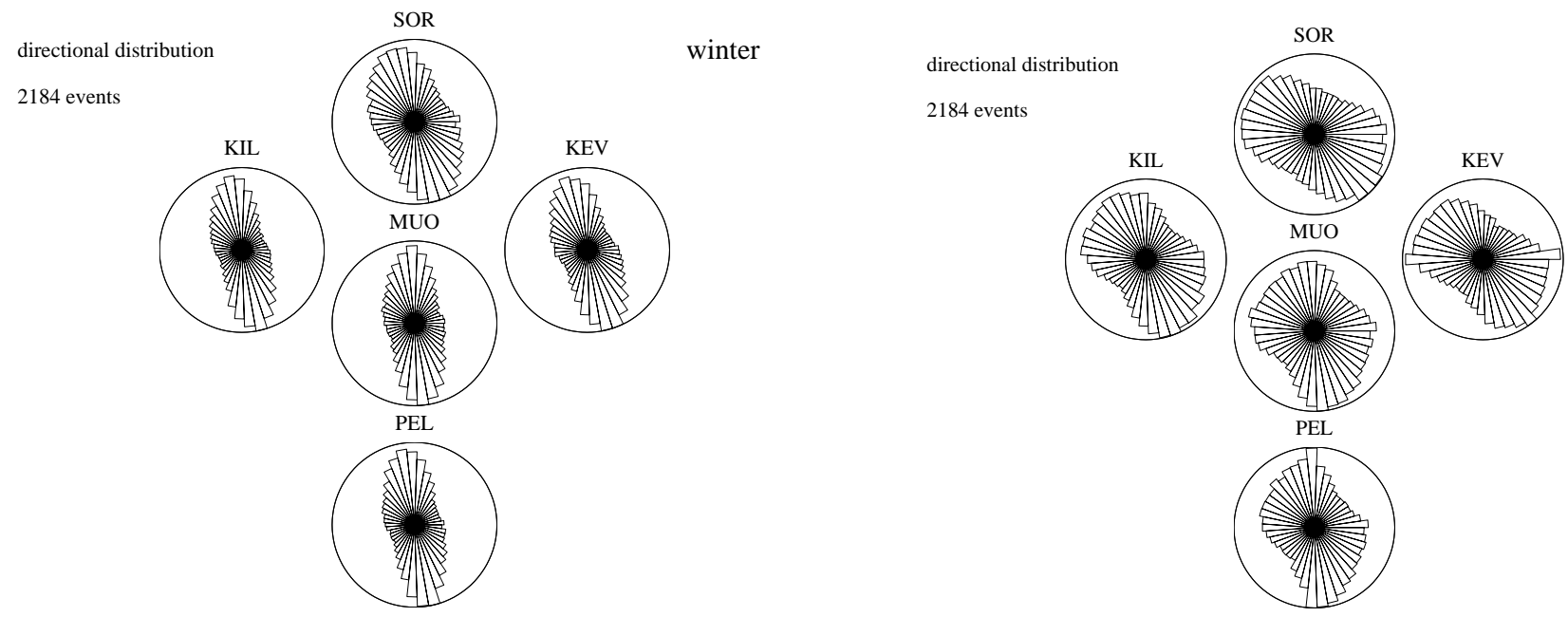

summer
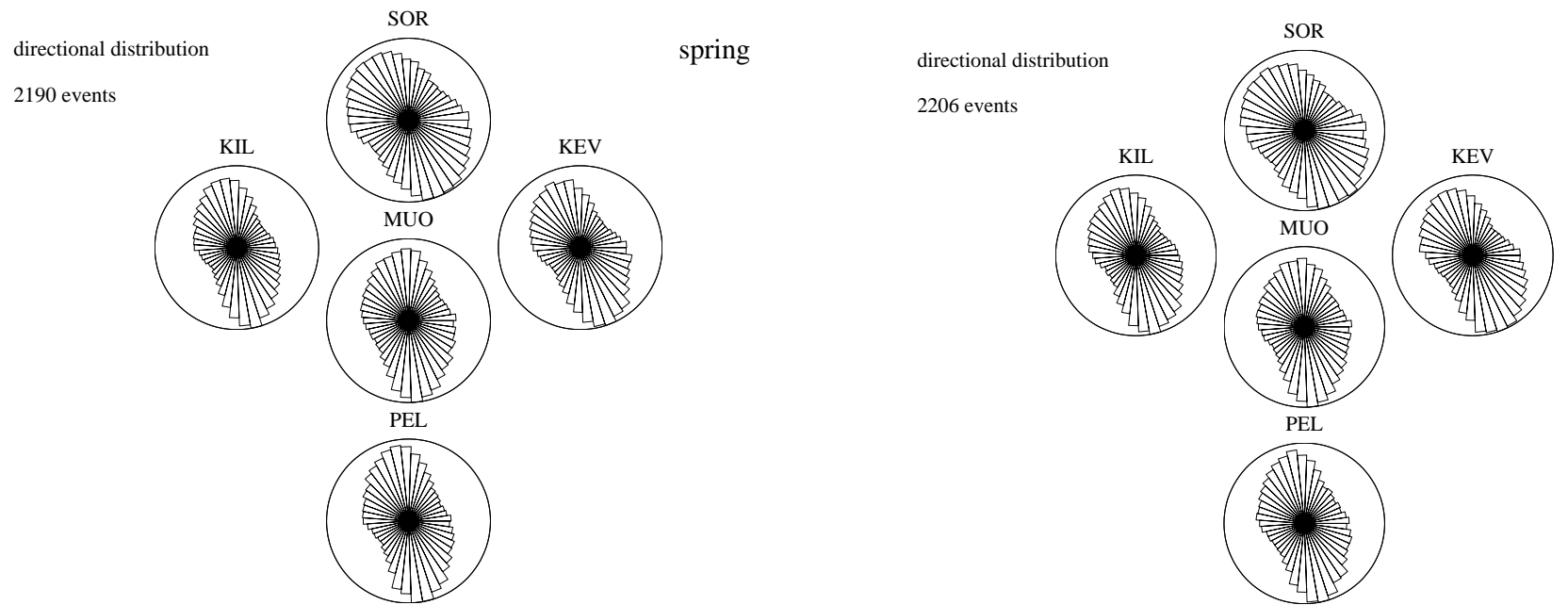

Fig. 3. Seasonal directional distributions of $d \boldsymbol{H} / d t$ exceeding $1 \mathrm{nT} \mathrm{s}{ }^{-1}$ at the five stations in Table 1 during the solar cycles $22-23$ (19862009). Autumn and spring refer to about 90 days centred at equinoxes, and summer and winter are similarly centred at solstices.

Table 2. Yearly number of high-speed streams (HSS) whose velocity exceeded $700 \mathrm{~km} \mathrm{~s}^{-1}$ and which lasted longer than $6 \mathrm{~h}$ (extended from Tanskanen et al., 2005).

\begin{tabular}{lrrrrrrrrr}
\hline Year & 1993 & 1994 & 1995 & 1996 & 1997 & 1998 & 1999 & 2000 & 2001 \\
HSS & 2 & 21 & 4 & 0 & 0 & 3 & 2 & 8 & 5 \\
\hline Year & 2002 & 2003 & 2004 & 2005 & 2006 & 2007 & 2008 & 2009 & 2010 \\
HSS & 7 & 27 & 3 & 13 & 1 & 0 & 2 & 0 & 1 \\
\hline
\end{tabular}

calculated monthly averages (lower panel of Fig. 7). The westward electrojet has a clear seasonal variation with spring and autumn maximum. The eastward electrojet in turn has a single maximum in summer. This behaviour is expected, since a corresponding seasonal variation is known for the global AU and AL indices (Ahn et al., 2000). Since large $d \boldsymbol{H} / d t$ values are mostly related to westward currents, their occurrence has a similar seasonal variation.

\section{Summary and discussion}

This paper confirms and specifies several previous findings of the behaviour of large $d \boldsymbol{H} / d t$ (exceeding $1 \mathrm{nT} \mathrm{s}^{-1}$ ) at high latitudes:

1. $d \boldsymbol{H} / d t$ activity is high around the midnight and early morning hours, and nearly vanishes at noon and early afternoon. This behaviour takes place during all 

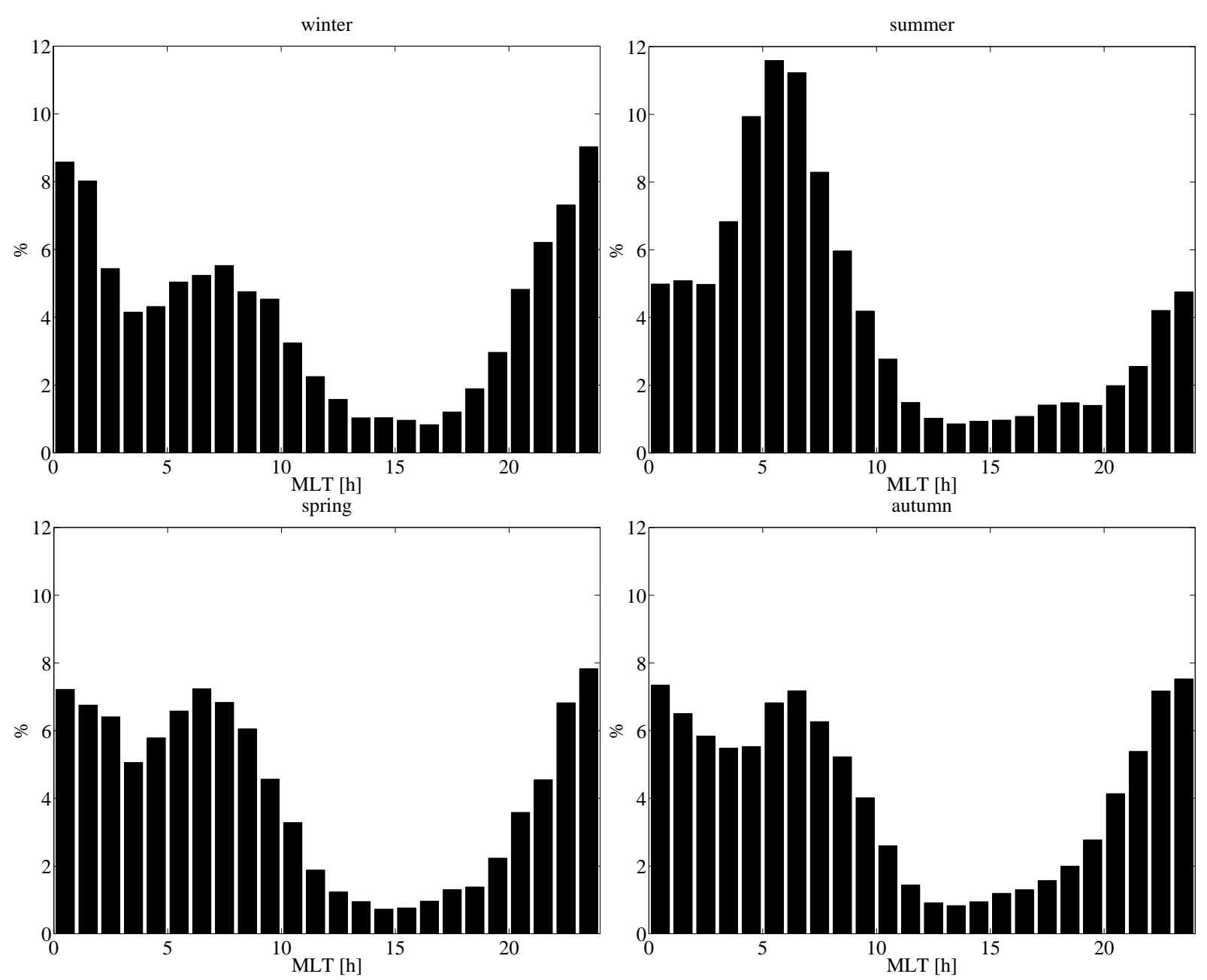

Fig. 4. Average diurnal occurrence of $d \boldsymbol{H} / d t$ exceeding $1 \mathrm{nT} \mathrm{s}^{-}$at five stations in northern Europe (Table 1) over the solar cycles 22-23 in 1986-2009 during different seasons.

seasons, although the midnight maximum is nearly invisible during summer.

2. Large $d \boldsymbol{H} / d t$ values occur predominantly during westward electrojets associated with southward oriented $\boldsymbol{H}$ vectors. Before and around midnight, $d \boldsymbol{H} / d t$ tends to be north-south oriented (parallel or opposite to $\boldsymbol{H}$ ). However, in the morning hours, the direction of $d \boldsymbol{H} / d t$ may differ tens of degrees from the north-south direction.

3. $d \boldsymbol{H} / d t$ activity is highest during spring and autumn and lowest during summer. $d \boldsymbol{H} / d t$ tends to be more northsouth oriented during winter than other seasons. The largest scatter in directions occurs during summer, when most events occur during the morning hours.

4. The yearly fraction of east-west directed large $d \boldsymbol{H} / d t$ vectors at the Kilpisjärvi station (MLAT 65.88) varies from 31 to $47 \%$ without any clear correlation with the general geomagnetic activity. It has no evident simple connection to the yearly averages of solar wind parameters either.

The basic feature of the diurnal behaviour of large $d \boldsymbol{H} / d t$ is evidently well-understood. Before and around midnight, a large-scale westward electrojet is dominating. It produces a southward $\boldsymbol{H}$ and a northward or southward $d \boldsymbol{H} / d t$ depending on whether the amplitude of the electrojet is decreasing or increasing. In the early morning, there is still a strong westward electrojet in the background, but also smaller-scale but rapidly changing structures such as vortices obviously related to geomagnetic pulsations. In such conditions, $d \boldsymbol{H} / d t$ can easily have any orientation.

Although the main characteristics of large $d \boldsymbol{H} / d t$ seem to prevail from one solar cycle to another, we still lack a deeper understanding of their origin. It is obvious that a physical explanation will require consideration of the whole geospace environment from the solar wind through the magnetosphere to the ionosphere. The relative importance of each region as 


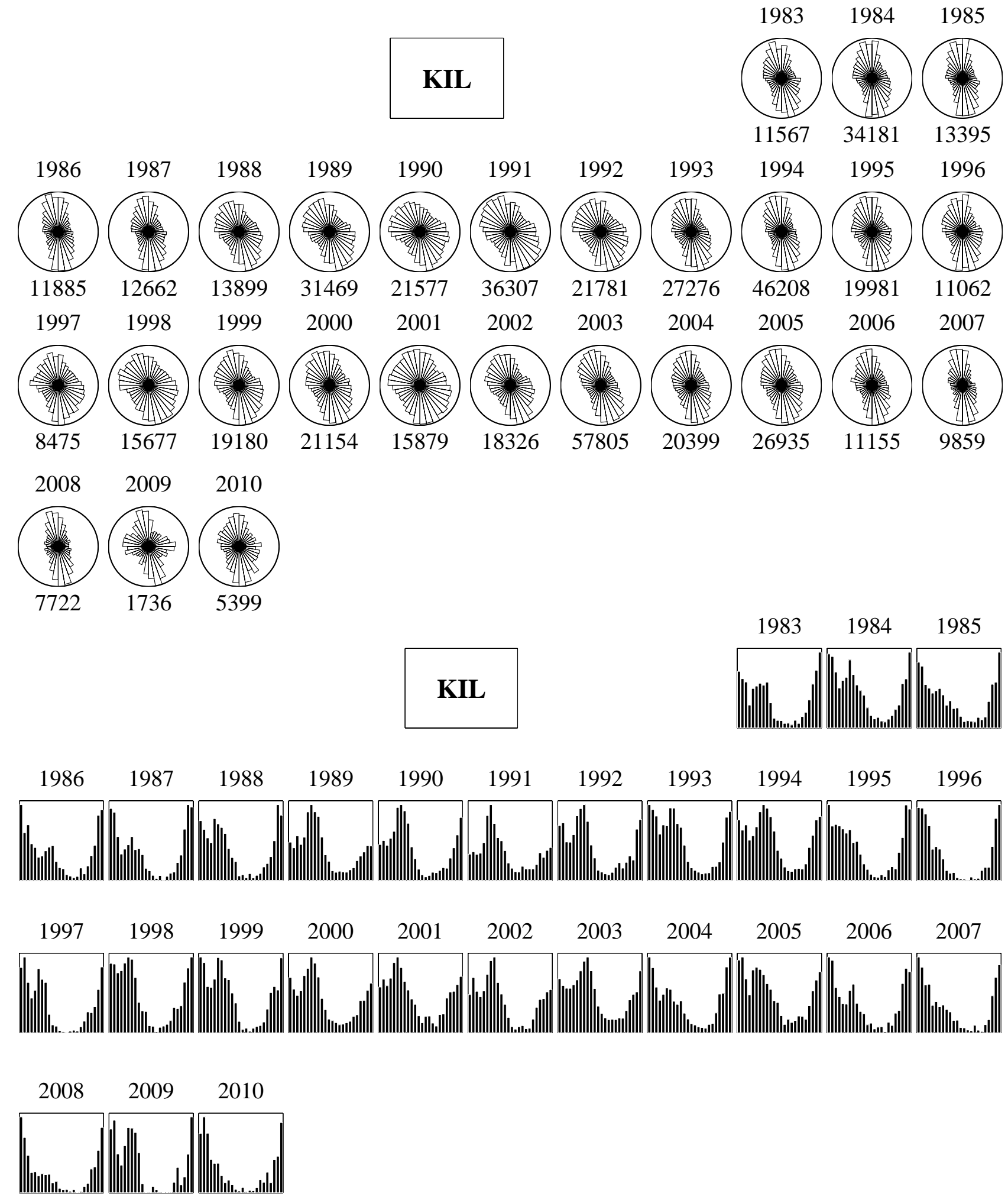

Fig. 5. Upper part: Directional distributions of $d \boldsymbol{H} / d t$ exceeding $1 \mathrm{nT} \mathrm{s}{ }^{-1}$ at KIL. The number of values is given below each yearly plot (due to occasional data gaps, these numbers are not completely comparable). Yearly diagrams are arranged so that the approximate start of each solar cycle is on the left-hand side. Lower part: The normalised diurnal occurrence of large $d \boldsymbol{H} / d t$ values as a function of MLT (00:00-24:00). 

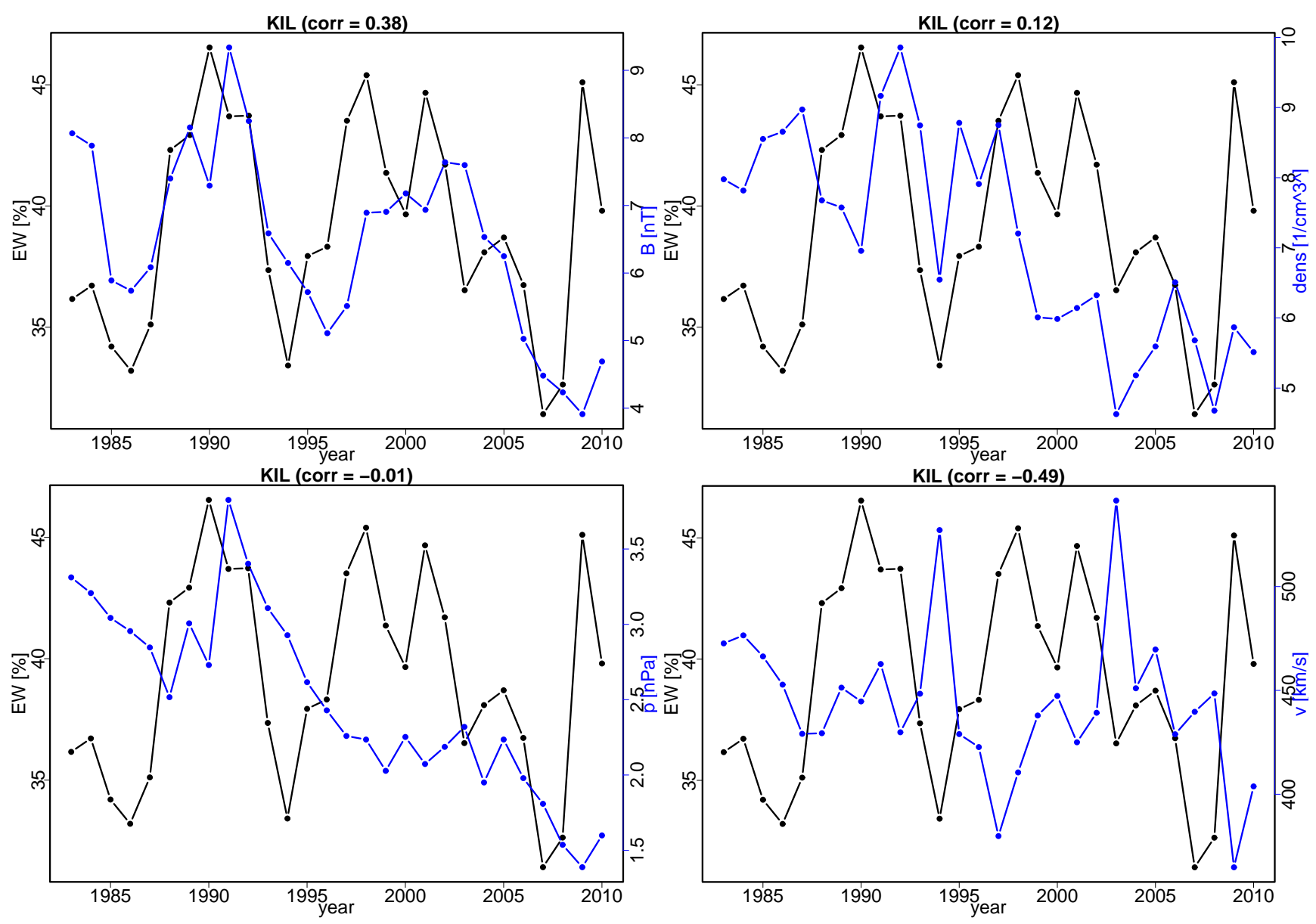

Fig. 6. Yearly fraction of EW directed large $d \boldsymbol{H} / d t$ vectors at KIL (black) and the following solar wind parameters (blue): magnitude of the magnetic field $(B)$, density $($ dens $)$, dynamic pressure $(p)$, velocity $(v)$.

well as of the related phenomena is unknown. We list and discuss here some specific items:

1. Concerning the solar wind and magnetospheric background, we know that coronal mass ejections (CME) are the major cause of large GIC, or practically equivalently of large $d \boldsymbol{H} / d t$ (Borovsky and Denton, 2006; Huttunen et al., 2008). Huttunen et al. (2008) examined the effectiveness of different structures embedded in a CME (sheath regions, ejecta, and boundary layers) in causing large GIC. When a CME interacts with the magnetosphere, the most intense GIC activity is likely to take place during the passage of the turbulent sheath region. The effectiveness of sheath regions in driving large GIC is possibly due to their capability to trigger substorms and to drive intense directly driven ionospheric activity. Ejecta-associated GIC seem to require an on-going magnetospheric Dst storm, while sheath regions and boundary layers can cause large GIC even when no activity is taking place in terms of Dst.
2. Kataoka and Pulkkinen (2008) showed that GIC amplitudes in the Finnish natural gas pipeline are relatively small during corotating interacting region (CIR) storms in comparison to CME storms. However, Pc3-5 pulsation activity during CIR storms drives long-lasting GIC in the local prenoon sector. Despite of the differences between CME and CIR storms, the maximum hourly value of $d \boldsymbol{H} / d t$ is always an excellent indicator for the maximum hourly amplitude of GIC in the Finnish pipeline for any local time and any storm phase of CME/CIR storms. Although Kataoka and Pulkkinen (2008) did not study auroral latitudes, we may speculate a similar behaviour there. Yagova et al. (2010) consider spectral parameters of high latitude geomagnetic disturbances in the Pc5/Pi3 frequency range (periods of about $250-1000$ s). Their Fig. 3 shows a nighttime maximum of the spectral power at CGM latitudes 65-67 partly overlapping the region of the five stations in Table 1. Furthermore, their Fig. 7 indicates an enhanced occurrence of high frequency variations in morning hours (06:00-12:00 MLT) at the same time when large $d \boldsymbol{H} / d t$ 

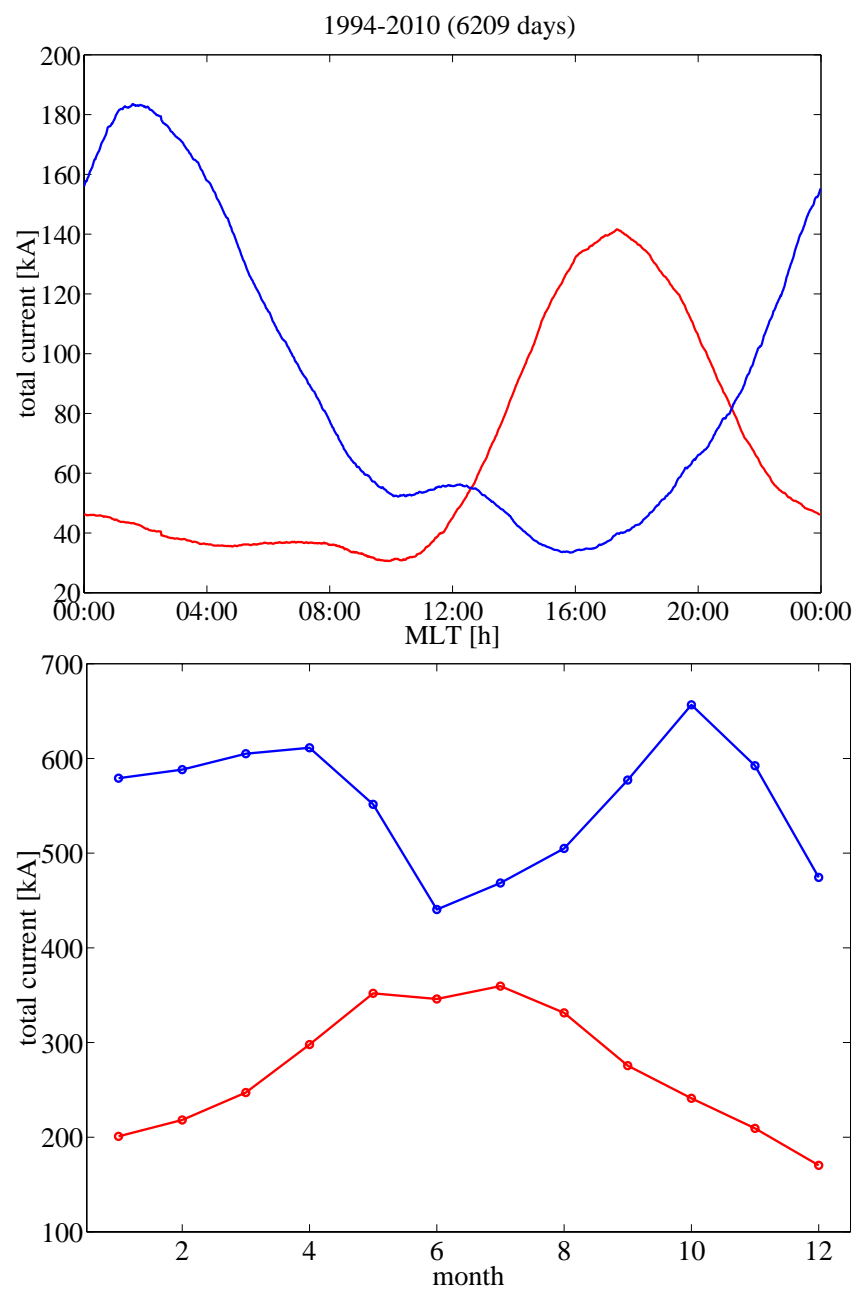

Fig. 7. Characteristics of ionospheric equivalent currents in 19942010 across the geographic meridian $22.1^{\circ} \mathrm{E}$ in the geographic latitude range of $59.0-79.4^{\circ} \mathrm{N}$. Upper panel: Average diurnal variation of the total eastward (red) and westward (blue) currents. Lower panel: Monthly averages of the daily maximum amplitudes.

occur frequently (cf. our Fig. 4). Since large $d \boldsymbol{H} / d t$ values are obviously related to high frequencies, the results by Yagova et al. (2010) at least qualitatively support our findings from a different viewpoint.

3. Weigel et al. (2003) studied separately different solar wind parameters to estimate how much influence they have on explaining variations of the northward magnetic field and its time derivative ( $X$ and $d X / d t$ ) in the region of IMAGE magnetometers. They found that the process that drives $X$ is different from that which drives $d X / d t$. For $X$, the solar wind velocity $v$ and IMF $B_{\mathrm{Z}}$ are the most important drivers when used as model inputs: they explain most of the variance of the $X$ time series. For $d X / d t$, the form of the coupling is sensitive to the local time, which is evident also from our Fig. 1 showing the large variability of the preferred orientation of $d \boldsymbol{H} / d t$. At 10:00 MLT, $v$ has the greatest influence and IMF $B_{\mathrm{Z}}$ has nearly no influence on the prediction efficiency. However, in the postmidnight sector IMF $B_{\mathrm{Z}}$ explains a substantial portion of the $d X / d t$ variance while $v$ has a much smaller role. The solar wind density was found to have an insignificant effect in agreement with our Fig. 6.

4. During the most active high-speed stream years (1994, 2003), a high solar wind velocity $v$ is related to a less scattered $d \boldsymbol{H} / d t$. We could intuitively expect more scatter during more disturbed periods when ionospheric currents might have a more complicated structure. However, there seems to be no such simple straightforward relationship. Furthermore, the smallest scatter occurs during a quiet year in 2007 with a typical yearly mean of $v$.

5. Large $d \boldsymbol{H} / d t$ values occur in the nighttime, so they are evidently closely connected to substorm activity. However, there are seasonal differences in the diurnal occurrence of large $d \boldsymbol{H} / d t$, especially when summer is compared to other seasons. Compared to the substorm occurrence, their number and intensity in summer is smaller than at other times, but on the other hand they last longer (Tanskanen, 2009). An initial study shows that summer substorms start $0.5-1 \mathrm{~h}$ later, but this does not explain the lack of $d \boldsymbol{H} / d t$ maximum at midnight.

Acknowledgements. The work of E.T. was supported by the Academy of Finland (projects no. 108518 and 128632). We appreciate very much the long-term careful work by several institutes who have operated the IMAGE magnetometer network. The solar wind OMNI2 data were obtained from the GSFC/SPDF OMNIWeb interface at http://omniweb.gsfc.nasa.gov/.

Topical Editor P.-L. Blelly thanks V. Pilipenko and another anonymous referee for their help in evaluating this paper.

\section{References}

Ahn, B.-H., Kroehl, H. W., Kamide, Y., and Kihn, E. A.: Seasonal and solar cycle variations of the auroral electrojet indices, J. Atmos. Sol.-Terr. Phys., 62, 1301-1310, 2000.

Amm, O. and Viljanen, A.: Ionospheric disturbance magnetic field continuation from the ground to the ionosphere using spherical elementary current systems, Earth Planets Space, 51, 431-440, 1999.

Borovsky, J. E. and Denton, M. H.: Differences between CMEdriven storms and CIR-driven storms, J. Geophys. Res., 111, A07S08, doi:10.1029/2005JA011447, 2006.

Huttunen, K. E. J., Kilpua, S. P., Pulkkinen, A., Viljanen, A., and Tanskanen, E.: Solar wind drivers of large geomagnetically induced currents during the solar cycle 23, Space Weather, 6, S10002, doi:10.1029/2007SW000374, 2008.

Kataoka, R. and Pulkkinen, A.: Geomagnetically induced currents during intense storms driven by coronal mass ejections and corotating interacting regions, J. Geophys. Res., 113, A03S12, doi:10.1029/2007JA012487, 2008. 
Minamoto, Y. and Taguchi, Y.: Significant decreases in the geomagnetic indices in the ascending phase of Solar Cycle 24, Earth Planets Space, 61, e25-e28, 2009.

Pirjola, R.: Review of the calculation of surface electric and magnetic fields and of geomagnetically induced currents in groundbased technological systems, Surv. Geophys., 23, 71-90, 2002.

Pulkkinen, A., Amm, O., Viljanen, A., and BEAR Working Group: Ionospheric equivalent current distributions determined with the method of elementary current systems, J. Geophys. Res., 108, doi:10.1029/2001JA005085, 2003.

Pulkkinen, A., Klimas, A., Vassiliadis, D., Uritsky, V., and Tanskanen, E.: Spatiotemporal scaling properties of the ground geomagnetic field variations, J. Geophys. Res., 111, A03305, doi:10.1029/2005JA011294, 2006.

Pulkkinen, A., Hesse, M., Habib, S., Van der Zel, L., Damsky, B., Policelli, F., Fugate, D., Jacobs W., and Creamer, E.: Solar shield: forecasting and mitigating space weather effects on highvoltage power transmission systems, Nat. Hazards, 53, 333-345, doi:10.1007/s11069-009-9432-x, 2009.

Tanskanen, E. I.: A comprehensive high-throughput analysis of substorms observed by IMAGE magnetometer network: Years 1993-2003 examined, J. Geophys. Res., 114, A05204, doi:10.1029/2008JA013682, 2009.

Tanskanen, E. I., Viljanen, A., Pulkkinen, T. I., Pirjola, R., Häkkinen, L., Pulkkinen, A., and Amm, O.: At substorm onset, $40 \%$ of AL comes from underground, J. Geophys. Res., 106, 13119-13134, 2001.

Tanskanen, E. I., Slavin, J. A., Tanskanen, A. J., Viljanen, A., Pulkkinen, T. I., Koskinen, H. E. J., Pulkkinen, A., and Eastwood, J.: Magnetospheric substorms are strongly modulated by interplanetary high-speed streams, Geophys. Res. Lett., 32, L16104, doi:10.1029/2005GL023318, 2005.
Turnbull, K. L., Wild, J. A., Honary, F., Thomson, A. W. P., and McKay, A. J.: Characteristics of variations in the ground magnetic field during substorms at mid latitudes, Ann. Geophys., 27, 3421-3428, doi:10.5194/angeo-27-3421-2009, 2009.

Viljanen, A., Nevanlinna, H., Pajunpää, K., and Pulkkinen, A.: Time derivative of the horizontal geomagnetic field as an activity indicator, Ann. Geophys., 19, 1107-1118, doi:10.5194/angeo19-1107-2001, 2001.

Viljanen, A., Pulkkinen, A., Amm, O., Pirjola, R., Korja, T., and BEAR Working Group: Fast computation of the geoelectric field using the method of elementary current systems and planar Earth models, Ann. Geophys., 22, 101-113, doi:10.5194/angeo-22101-2004, 2004.

Viljanen, A., Tanskanen, E. I., and Pulkkinen, A.: Relation between substorm characteristics and rapid temporal variations of the ground magnetic field, Ann. Geophys., 24, 725-733, doi:10.5194/angeo-24-725-2006, 2006.

Watermann, J.: The magnetic environment - GIC and other ground effects, in: Space Weather, Research Towards Applications in Europe, edited by: Lilensten. J., Springer, 269-275, 2008.

Weigel, R. S., Klimas, A. J., and Vassiliadis, D.: Solar wind coupling to and predictability of ground magnetic fields and their time derivatives, J. Geophys. Res., 108, 1298, doi:10.1029/2002JA009627, 2003.

Yagova, N. V., Pilipenko, V. A., Baransky, L. N., and Engebretson, M. J.: Spatial distribution of spectral parameters of high latitude geomagnetic disturbances in the Pc5/Pi3 frequency range, Ann. Geophys., 28, 1761-1775, doi:10.5194/angeo-28-17612010, 2010. 